\title{
MOLECULAR IDENTIFICATION AND PHYLOGENTIC ANALYSIS OF POTATO LEAF ROLL VIRUS IN EGYPT
}

\author{
ABDELMAKSOUD, H. M., A. M. MANDOUR and A. SH. GAMAL ELDIN \\ Plant Virology Research Department, Plant Pathology Research Institute, ARC, Giza - Egypt.
}

(Manuscript received 19 March 2013)

\begin{abstract}
Potato leaf roll virus (PLRV) was isolated from Egyptian grown potatos. The virus was identified on the basis of host range, symptomatology, insect transmission, electron microscopy, RTPCR, and PCR-ELISA. The complete nucleotide sequence of genomic PLRV-RNA was obtained from cloned cDNA and submitted in GenBank under Accession No. AY138970. This sequence is 5884 nucleotide long and encodes 5 ORF with an unique read-through protein suggested a conflict with the conceptual translation at amino acid 209. Comparison of PLRV sequence with that of other PLRV strains shows overall similarities of $97.02 \%$ and high genetic identity of $100 \%$ (Polish Strain), 98.5\% (French Strain), 98.4\% (Wageningen Strain), 98.3\% (UK Strain), 98\% (Canadian Strain), and $93.5 \%$ (Australian Strain).

Keywords: Phylogentic analysis, PLRV similarities, Egypt PLRV
\end{abstract}

\section{INTRODUCTION}

PLRV is the most important aphid transmitted potato virus belonged to Luteovirus. The family Luteoviridae have long been recognized as a natural group they sharing biological characters and particle features, they also differ in molecular characteristics. The recent taxonomy creates a family Luteoviridae that contain 3 genera to accommodate this diversity Genus Luteovirus (Type species Barely yellow dwarf virus -PAV), Genus Pelerovirus (Type species Potato leaf roll virus), Genus Enamovirus (Type species Pea enation mosaic virus). PLRV particles is $25 \mathrm{~nm}$ in diameter, isometric and contain 5.3 to $9.5 \mathrm{~kb}$ ssRNA and a major $23 \mathrm{k}$ coat protein with a "read through" proteins of $60 \mathrm{k}$ to $90 \mathrm{k}$. The genomic comprise 5 or 6 large ORFs (Waterhouse et a/ 1988, and Gamal Eldin, A. S. et a/2004).

The aim of the present study was to compare the full genomic sequence of an Egyptian strain of PLRV with other PLRV strains from different countries and with other viruses belong to Luteoviridae using most up to date genetic software analysis DNASTAR Lasergene version 10 (DNASTAR Inc, MD). 


\section{MATERIALS AND METHODS}

\section{Virus isolation:-}

Leaf samples of potato (Solanum tuberosum) showing leaf roll symptoms were collected from the potato fields at the Kaluboiua Governorate and were for virus isolation. The samples were serologicaly tested using PLRV- PAbs ELISA Kit (LOEWE Biochemical $\mathrm{GmbH}$, Germany). The virus was transmitted by Myzus persicae Sulz using Physalis foloridana seedlings as a test plants and as a virus source for further studies.

\section{RNA purification and RT-PCR}

RNA was extracted by RNeasy Plant Mini Kit (Qiagene, Germany) as recommended by the manufacturer. After total RNA extraction, the PCR detection was carried out using QIAGEN OneStep RT-PCR Kit (Qiagene, Germany) utilized two PLRV-Specific pairs of primers. The foreword primer 5' AGCGCATAAACTCTACACTCATTG and the reverse primer $5^{\prime}$ GTATCCTTCCACAGCCCTCTCATT (metabion $\mathrm{GmbH}$, Germany) corresponding to positions 31-54 and 832-809 of PLRV genome. The PCR products were analyzed by gel electrophoresis on $1 \%$ agarose gel prepared in 1X TBE buffer (Sambrook et al 1989). The gel was stained with ethidium bromide and examined using UV transilluminater and the PCR fragments of PLRVwas confirmed as $802 \mathrm{bp}$.

\section{CDNA Library and PCR Cloning}

The entire PLRV-RNA CDNA libraries was constructed using First Strand CDNA Synthesis Kit (Promega, USA) and each represented library was ligated directly into TA cloning vector (Plasmid PCR ${ }^{\mathrm{TM}} \mathrm{II}$ ). The ligation products were transformed into competent of $E$. coli (INV\& $\mathrm{F}^{\prime}$ cells). White, ampicillin-resistant, colonies were selected and screened for correct inserts.

\section{DNA Sequencing}

The nucleotide sequence of PLRV clone liberary was determined by the method of Beck (1993). The sequence was performed with ALF DNA Sequencer based on an adaptation of Sanger dideoxy methodology (Sanger et al, 1977). The nucleotide sequence of PLRV was carried out at Molecular Virology Group-Biotechnology Group, Department of Plant Biology-The Royal Veterinary and Agricultural University (KVL), Copenhagen-Denmark. The PLRV sequence was computer translated with the program Fragment Manger Software (Amersham Inc) and further analyzed by DNASTAR Lasergene (DNASTAR Inc, MD). 


\section{RESULTS}

The complete genomic nucleotide sequence is shown in Fig (1). It contains 5884 bp. The base comparison of PLRV RNA $(22.37 \% \mathrm{U}, 25.24 \% \mathrm{C}, 27.88 \% \mathrm{~A}$ and $24.31 \mathrm{G})$, while $(A+T)=50.45$ and $(C+G)=49.55 \%$ and the base count was $1640 A$, $14850 \mathrm{C}, 1430 \mathrm{G}$, and $1328 \mathrm{U}$ and the predicated translation of the amino acids is shown in Fig (1), below each triplet.

Fig. 1. The complete nucleotide sequence of PLRV-Egypt and its predicted encoded amino acid sequence

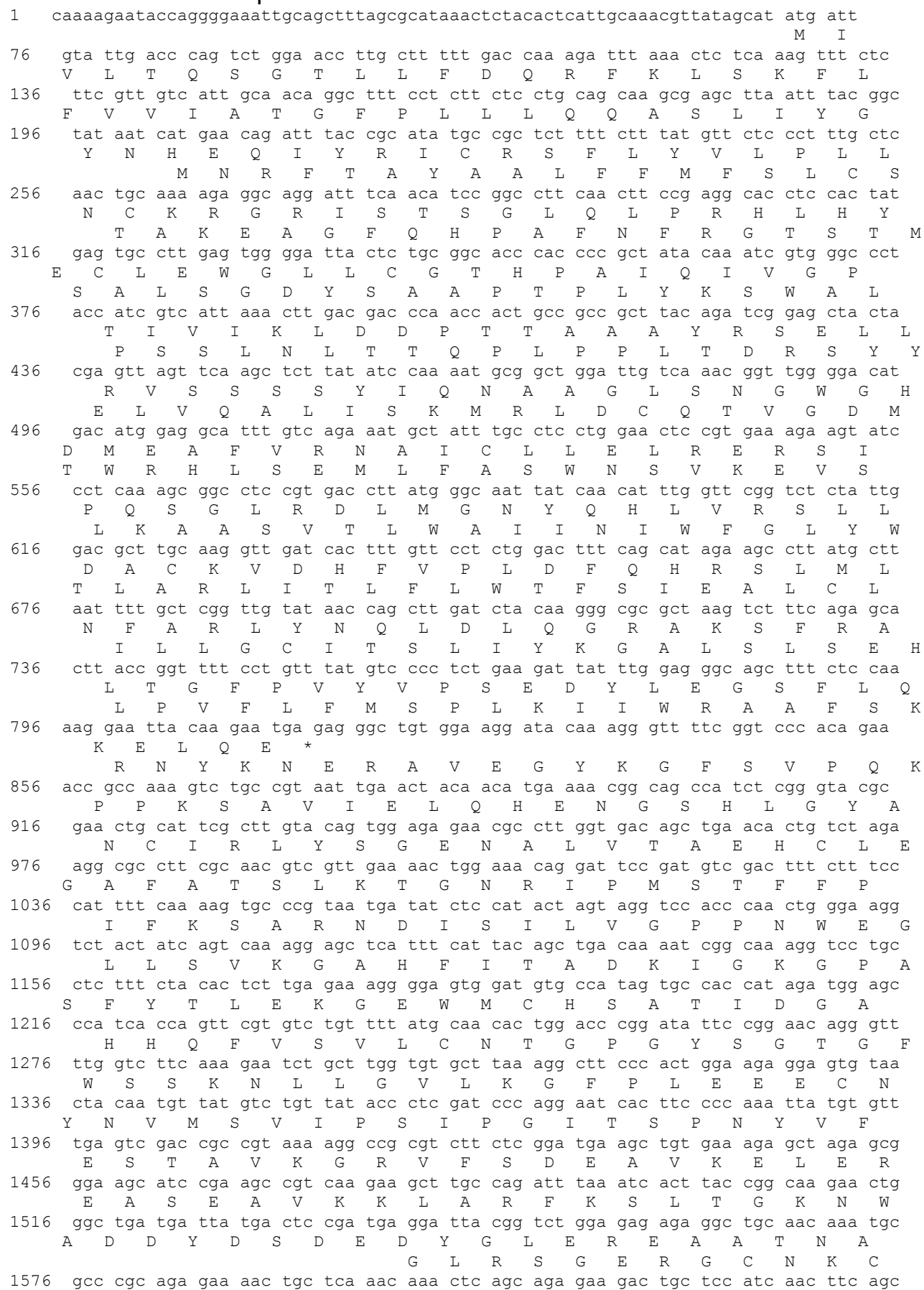




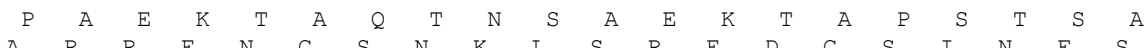

1636 aga gaa aac tgc tcc aac aaa caa gcc ttt aaa tgg gca agc ggc acc gtc cgc caa aac $\begin{array}{lllllllllllllllllllllllllll} & \mathrm{E} & \mathrm{K} & \mathrm{T} & \mathrm{A} & \mathrm{P} & \mathrm{T} & \mathrm{N} & \mathrm{K} & \mathrm{P} & \mathrm{L} & \mathrm{N} & \mathrm{G} & \mathrm{Q} & \mathrm{A} & \mathrm{A} & \mathrm{P} & \mathrm{S} & \mathrm{A} & \mathrm{K} & \mathrm{T}\end{array}$ $\begin{array}{lllllllllllllllllllllllllll}R & E & N & C & S & N & K & Q & A & F & K & W & A & S & G & T & V & R & Q & N\end{array}$

1696 aaa cgg caa ctc cga cat ccc cga cgt cgc tac aag cgc acc acc aat gga caa aat ggt $\begin{array}{llllllllllllllllllllllllllll}N & G & N & S & D & I & P & D & V & A & T & S & A & P & P & M & D & K & M & V \\ K & R & Q & L & R & H & P & R & R & R & Y & K & R & T & T & N & G & Q & N & G\end{array}$

1756 cga aca gat cat cac agc tat ggt ggg gag aat caa tct ctc gga gat aga gga gaa gat $\begin{array}{llllllllllllllllllllllllllllllll}E & Q & I & I & T & A & M & V & G & R & I & N & L & S & E & I & E & E & K & I\end{array}$

1816 agt gag cag ggt gtc tca gaa agc cct gca gaa gcc caa aca aaa gaa gcg cgg aag gcg

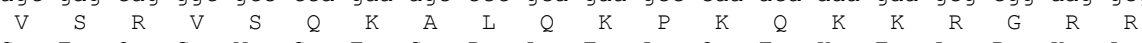
$\begin{array}{llllllllllllllllllllllllllllllllllll}S & E & Q & G & V & S & E & S & P & A & E & A & Q & T & K & E & A & R & K & A\end{array}$

1876 tgg agg gaa gaa caa gca aaa cag ttc acc tcc tac ttc aac gca atc tac aag tgg ggc $\begin{array}{lllllllllllllllllllllllllllllllll}G & G & K & N & K & Q & N & S & S & P & P & T & S & T & Q & S & T & S & G & A \\ W & R & E & E & Q & A & K & Q & F & T & S & Y & F & N & A & I & Y & K & W & G\end{array}$

1936 gcc caa gaa gaa ggc tgt ccc cca ggc ttc agg aag tgc ggg cac atc ccc ggc tac tac

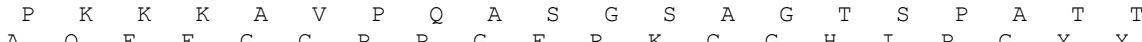

1996 cac ccc cgc acc aga ggc gaa acc cag tgg ggg caa aaa ctc tgc caa gtt cat ccc gag $\begin{array}{lllllllllllllllllllll}\mathrm{T} & \mathrm{P} & \mathrm{A} & \mathrm{P} & \mathrm{E} & \mathrm{A} & \mathrm{K} & \mathrm{P} & \mathrm{S} & \mathrm{G} & \mathrm{G} & \mathrm{K} & \mathrm{N} & \mathrm{S} & \mathrm{A} & \mathrm{K} & \mathrm{F} & \mathrm{I} & \mathrm{P} & \mathrm{S}\end{array}$ $\begin{array}{lllllllllllllllllllll}H & P & R & T & R & G & E & T & Q & W & G & Q & K & L & C & Q & V & H & P & E\end{array}$

2056 ctg gcg gag aaa aca aca gga ttc ggc tgg cca aaa gcc gga tct gaa gct gaa ctc caa

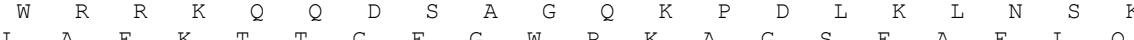

2116 agc ctg aat cta cag gct gcc agg tgg ctc caa cgc geg gag tcg gcc act atc cct ggc $\begin{array}{lllllllllllllllllllll}\mathrm{A} & { }^{*} & \mathrm{~L} & \mathrm{~N} & \mathrm{~L} & \mathrm{Q} & \mathrm{A} & \mathrm{A} & \mathrm{R} & \mathrm{W} & \mathrm{L} & \mathrm{Q} & \mathrm{R} & \mathrm{A} & \mathrm{E} & \mathrm{S} & \mathrm{A} & \mathrm{T} & \mathrm{I} & \mathrm{P} & \mathrm{G}\end{array}$

2176 gca gaa gca aga aag cgc gtg att gag aaa aca gtg gag gca tac aga aat tgt gta act

$\mathrm{A}$
2236
aac gcc N A P L C S L K S K L D W A G F Q Q D I

2296 cgt gaa gca gtc cag tcc ctt gag cta gac get ggt gta ggc att ccc tat atc gcg tat

R A V O S L E I D A G V G I P Y I A Y

2356 ggc ctc ccc aca cac cga gga tgg gtt gag gac cat aag ctt ctc cca gtg ctc act cag

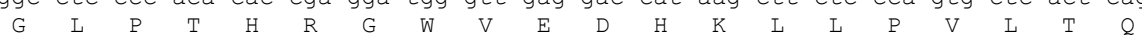

2416 ctg acc ttt gac cga cta cag aag atg tcg gag gcc agc ttt gag gat atg agc gca gaa $\begin{array}{llllllllllllllllllll}\mathrm{L} & \mathrm{T} & \mathrm{F} & \mathrm{D} & \mathrm{R} & \mathrm{L} & \mathrm{Q} & \mathrm{K} & \mathrm{M} & \mathrm{S} & \mathrm{E} & \mathrm{A} & \mathrm{S} & \mathrm{F} & \mathrm{E} & \mathrm{D} & \mathrm{M} & \mathrm{S} & \mathrm{A} & \mathrm{E}\end{array}$

2476 gag ctg gtt caa gaa ggg ctc tgt gat cct atc aga cta ttt gtc aaa gga gag ccc cac

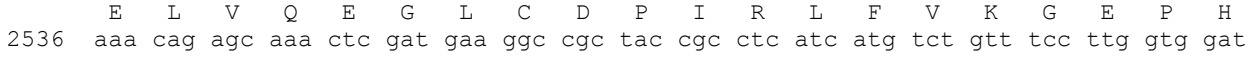

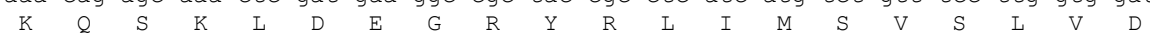

2596 caa ctg gta gcc cgg gtt ctg ttc caa aat cag aac aaa agg gaa att tcc ctg tgg agg $\begin{array}{lllllllllllllllllllll}Q & \mathrm{~L} & \mathrm{~V} & \mathrm{~A} & \mathrm{R} & \mathrm{V} & \mathrm{L} & \mathrm{F} & \mathrm{Q} & \mathrm{N} & \mathrm{Q} & \mathrm{N} & \mathrm{K} & \mathrm{R} & \mathrm{E} & \mathrm{I} & \mathrm{S} & \mathrm{L} & \mathrm{W} & \mathrm{R}\end{array}$

2656 tct gtg cet tcc aaa ccc ggt ttt ggc ctt tca act gac act caa act gct gaa ttc ttg $\begin{array}{lllllllllllllllllllllllllllll}S & V & P & S & K & P & G & F & G & L & S & T & D & T & Q & T & A & E & F & L\end{array}$

2716 gag tgt ctt caa aag gtg tct gga gcg cca tct gtg gaa gaa ttg tgt gca aat cac aag $\begin{array}{llllllllllllllllllll}E & C & L & Q & K & V & S & G & A & P & S & V & E & E & L & C & A & N & H & K\end{array}$

2776 gag tac acg cgc cca acc gac tgt tcc ggt ttc gac tgg tca gtc gcg tat tgg atg ctg $\begin{array}{llllllllllllllllllllllllll}E & Y & T & R & P & T & D & C & S & G & F & D & W & S & \text { V } & \text { A } & \text { Y } & \text { W } & M & \text { L }\end{array}$

2836 gag gat gat atg gag gtg aga aat cgc ctg aca ttt aat aac acc cag ctc acc aag cgc $\begin{array}{llllllllllllllllllllllll}E & D & D & M & E & V & R & N & R & L & T & F & N & N & T & Q & L & T & K & R\end{array}$

2896 ctt cgg gcc gcc tgg ttg aag tgc ata gga aat tcc gtc ctg tgc ctg tcc gat ggc act

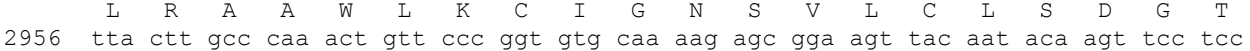

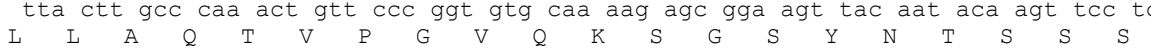

3016 aac tct aga atc cgg gtt atg gct gcc tat cac tgt ggc gcc gac tgg gca atg gcc atg

$\begin{array}{lllllllllllllllllllll}N & S & R & I & R & V & M & A & A & Y & H & C & G & A & D & W & A & M & A & M\end{array}$

3076 ggg gac gat get ctc gaa gcc CCC aac tcc gac ctg gag gag tat aaa aca cta ggt ttc

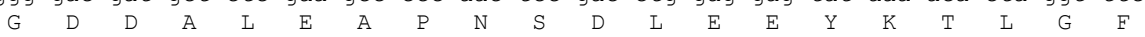

3136 aaa gtc gag gta ggt cga gaa ctc gaa ttc tgt tca cac atc ttc aga aat ccg acc ctc

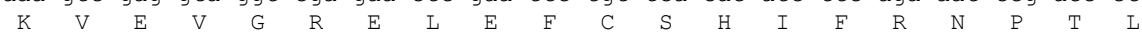

3196 gcc gtt ccg gtc aac acc aac aaa atg ctt tac aag ttg atc cat ggt tat aat ccg gaa $\begin{array}{llllllllllllllllllll}\mathrm{A} & \mathrm{V} & \mathrm{P} & \mathrm{V} & \mathrm{N} & \mathrm{T} & \mathrm{N} & \mathrm{K} & \mathrm{M} & \mathrm{L} & \mathrm{Y} & \mathrm{K} & \mathrm{L} & \mathrm{I} & \mathrm{H} & \mathrm{G} & \mathrm{Y} & \mathrm{N} & \mathrm{P} & \mathrm{E}\end{array}$ 3256 tgt ggc aat cca gaa gtg att caa aac tat ctg gct gca gta ttc tct gtg ctg cag gaa

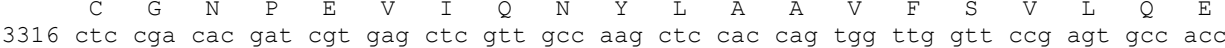
$\begin{array}{llllllllllllllllllll}\mathrm{L} & \mathrm{R} & \mathrm{H} & \mathrm{D} & \mathrm{R} & \mathrm{E} & \mathrm{L} & \mathrm{V} & \mathrm{A} & \mathrm{K} & \mathrm{L} & \mathrm{H} & \mathrm{O} & \mathrm{W} & \mathrm{L} & \mathrm{V} & \mathrm{P} & \mathrm{S} & \mathrm{A} & \mathrm{T}\end{array}$ 3376 aca aaa gaa cac tga agg agc tca cta aaa cta gcc aag cat acg cga gtt gca agc att 3436 gga agt tca agt ctc gtt aca tca acc gga caa aat aga tta taa att ttt agc ggg att

3496 tgc ttt agg att ctc atc cgc aat $\mathrm{ccc}$ att ttc agt agc cgg ttt ata ttt tgt tta cct 3556 aaa gat ttc ctc cca cgt gcg atc aat tgt taa tga gta cgg tcg tgg tta aag gaa atg
$\mathrm{M} \mathrm{S}_{\mathrm{T}} \mathrm{V}$ V V $\mathrm{K}$ G N V

3616 tca atg gtg gtg tac aac aac caa gaa ggc gaa gaa ggc aat ccc ttc gca ggc gcg cta

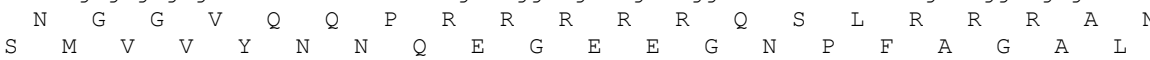

3676 aca gag ttc agc cgg tgg tta tgg tca cgg ccc ctg ggc aac cca ggc gtc gaa gac gca

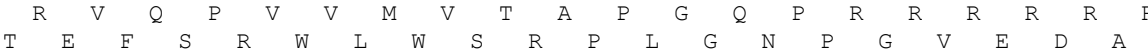


3736 gaa gag gag gca atc gcc gct caa gaa gaa ctg gag ttc ccc gag gac gag gct caa gcg $\begin{array}{lllllllllllllllllllll}R & G & G & N & R & R & S & R & R & T & G & V & P & R & G & R & G & S & S & E\end{array}$

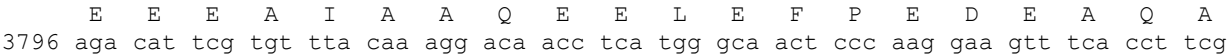

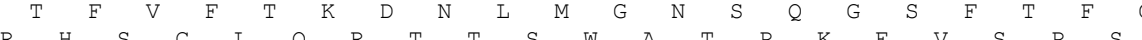
3856 ggc cga gtc tat cag act gtc cgg cat tca agg atg gaa tac tca agg cet acc atg agt

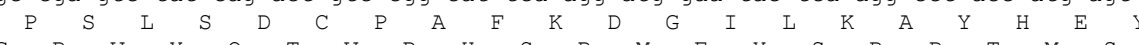

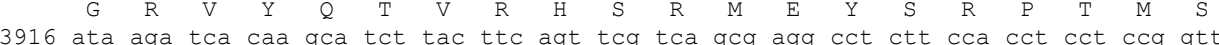
$\begin{array}{llllllllllllllllllll}K & I & T & S & I & L & L & Q & F & V & S & E & A & S & S & T & S & S & G & S\end{array}$ 3976 cca tcg ctt atg agt tgg acc ccc att gca aag tat cat ccc tcc agt cct acg tca aca

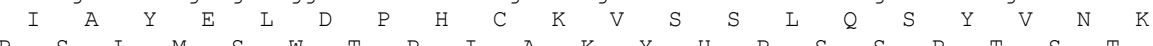

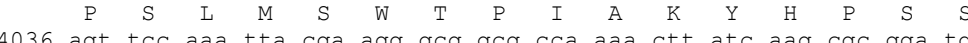

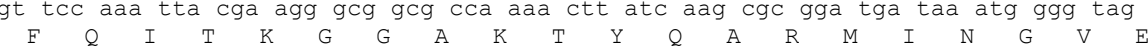

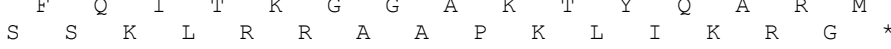

4096 aat ggc acg att ctt $c t g$ agg atc agt gcc gga tac tgt gga aag gaa atg gaa aat ctt

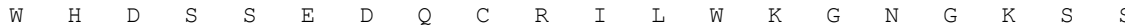
4156 cag ata ccg cag gat cet tca gag tca cca tca ggg tgg ctt tgc aaa acc cca aat agg $\begin{array}{lllllllllllllllllllllllllllllllll}D & T & A & G & S & F & R & V & T & I & R & V & A & L & Q & N & P & K & X & V\end{array}$ 4216 tag act ccg gat cag agc ctg gtc caa gcc cac a ac caa cac cca ctc caa ctc ccc aga 4276 agc acg agc gat tta ttg ctt acg ttg gca tac cta tgc taa cca ttc agg cca ggg aga 4336 acg acg acc aga tca tat tgg gtt ctt tag gga gcc aaa gga tga aat ata tag agg acg D D Q I I L G S L G S Q R M K Y I E D E 4396 aga acc aga act aca caa atg tta gtt ctg agt att act ctc aat cga gca tgc aag ccg 4456 tcc cta tgt att act tta atg tcc cga aag ggc aat ggt cag tcg aca tca gct gcg aag $\begin{array}{lllllllllllllllllllll}P & M & Y & Y & F & N & V & P & K & G & Q & W & S & V & D & I & S & C & E & G\end{array}$ 4516 ggt atc aac cca cta gca gca cct cgg atc caa acc ggg gta gga gtg acg ggg tga tcg 4576 cgt att caa acg cgg act ccg att att gga atg ttg gtg aag cgg atg gtg tca aaa ttt

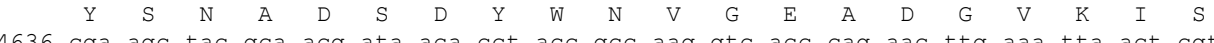
4636 cga agc tac gca acg ata aca cct acc gcc aag gtc acc cag aac ttg aaa tta act cgt $\begin{array}{cccccccccccccccccccc}\text { K } & \text { L } & R & N & D & N & T & Y & R & Q & G & H & P & E & L & E & I & N & S & C \\ 4696 & \text { gtc } & \text { att } & \text { ttc } & \text { gcg } & \text { agg } & \text { gCc } & \text { aac } & \text { tcc } & \text { ttg } & \text { aac } & \text { ggg } & \text { acg } & \text { cta } & \text { cala tta } & \text { gct } & \text { tcc } & \text { acg } & \text { ttg aag }\end{array}$

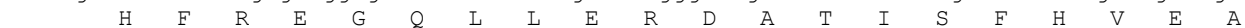
4756 cgc cta ctg atg ggc gat tct ttc tcg ttg gtc ccg cta tcc aga aaa ccg caa agt ata

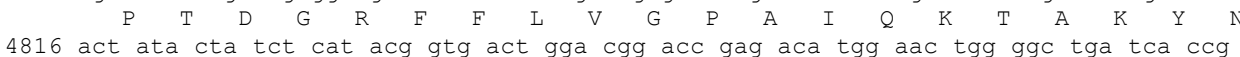
Y T I S Y G D W T D R D M E L G L I T V 4876 tgg tgc ttg atg aac att tag aag gca ctg gtt cgg cta aca gag tgc ggc ggc ccc cac 4936 ggg agg gcc aca cct ata tgg cct cgc cgc gcg aac cgg aag gaa aac cgg ttg gaa ata E G H T Y M A S P R E P E G K P V G N K 4996 aac caa ggg acg aaa ccc cga tac aaa cgc agg aaa gac aac ctg atc aaa ctc cgt ctg 5056 acg acg tat ccg atg ctg gtt cgg taa aca aca gcg gct caa ctg agt cgc tgc aat tgg D V S D A G S V N N S G S T E S L Q L E 5116 agt tcg ggg taa act cag ata gta cct acg atg cta cag tcg atg gta cag act ggc cca 5176 gaa ttc ctc cac caa ggc acc cac ctg aac cta gag ttt ccg gca att caa gaa ctg tta $\begin{array}{lllllllllllllllllllll}\mathrm{I} & \mathrm{P} & \mathrm{P} & \mathrm{P} & \mathrm{R} & \mathrm{H} & \mathrm{P} & \mathrm{P} & \mathrm{E} & \mathrm{P} & \mathrm{R} & \mathrm{V} & \mathrm{S} & \mathrm{G} & \mathrm{N} & \mathrm{S} & \mathrm{R} & \mathrm{T} & \mathrm{V} & \mathrm{l}\end{array}$ 5236 ttg act tte ctc cga aag ccg atc tat tgg aga att ggg atg ccg aac act tcg acc ctg 5296 gtt att cca aag aag atg tcg ctg ctg cta cta tta tag cgc acg gca gta ttc aag atg 5356 ggc gaa gta tgt tgg aga aga gag agg aaa gtg tca aga aca aaa cet cet cct gga agc R S M L E K R E E S V K N K T S S W K 5416 ccc cot tat cta aag cgg tga gcc cag cca tag cca aat tgc get cga ttc gca aat ccc

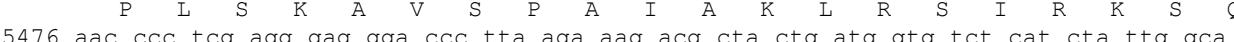
P L E G G T L K K D A T D G V S S I G S 5536 gtg gtt ctc taa cag gtg gca cgc tta aga gga agg taa cta ttg aag agc gtt tac tgc

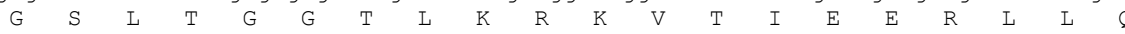
5596 aga cet taa caa ctg aac aaa ggc tgt ggt acg aga att tga aga aaa cta acc ctc cag

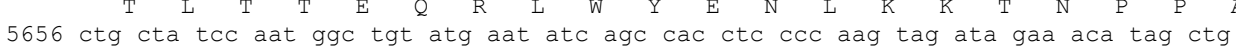

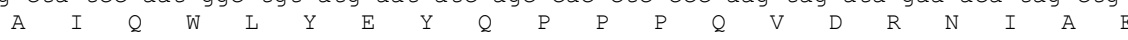
5716 aaa agc cat tcc aag gga gga aat gag tcg act cac gac tta aaa ctg agt gtc cgc cgg 5776 aca tta agc gga acg aaa gcc gaa agg tga tta ggc tct caa cgc ctg cta gag acc gtc

Percentage of different bases are (23\% U, 25\% C, $28 \% \mathrm{~A}$ and $24 \% \mathrm{G})$. These results are almost similar those of BWYV RNA and BYDV RNA (22\% U, 24\% C, 29.5\% 
A and $24.5 \% \mathrm{G})$. The Egyptian strain was compared with six PLRV sequences and the overall similarities for all genomic sequences were 100\%, 98.5\%, 98.4\%, 98.3\%, $98 \%$ and $93.5 \%$ for polish, French, Wageningen, UK, Canada, and Australian strains, respectively as shown in \{Table 1 and Fig 2\}. Five substantial open reading frames (ORFs) are present in PLRV RNA (Fig 2). ORF1 started from nucleotide 70 till nucleotide 813 (AA1-247), ORF2 from 203 to 2122 (AA1-639), ORF3 from 1540 to 3390 (AA 1-617), ORF 4 from 3588 to 5741 (AA1-718), while ORF5 started at 3613 and ended at 4083 (AA1-156). The overall similarities between Egyptian strain and other strains in the intergenic regions are shown in \{Table 2 and Fig 3\}.

Table 1. Overall percent identity of PLRV- $\mathrm{N}$ with different PLRV isolates

\begin{tabular}{|l|c|}
\hline \multicolumn{1}{|c|}{ Strain } & Similarity \% \\
\hline Egypt X Australia & 93.5 \\
\hline Egypt X UK & 98.3 \\
\hline Egypt X Polish & 100 \\
\hline Egypt X French & 98.5 \\
\hline Egypt X Canada & 98 \\
\hline Egypt X Wageningen & 98.4 \\
\hline
\end{tabular}

Fig. 2. Phylogenetic tree determines dimensions of relationship between Egyptian PLRV and different PLRV strains

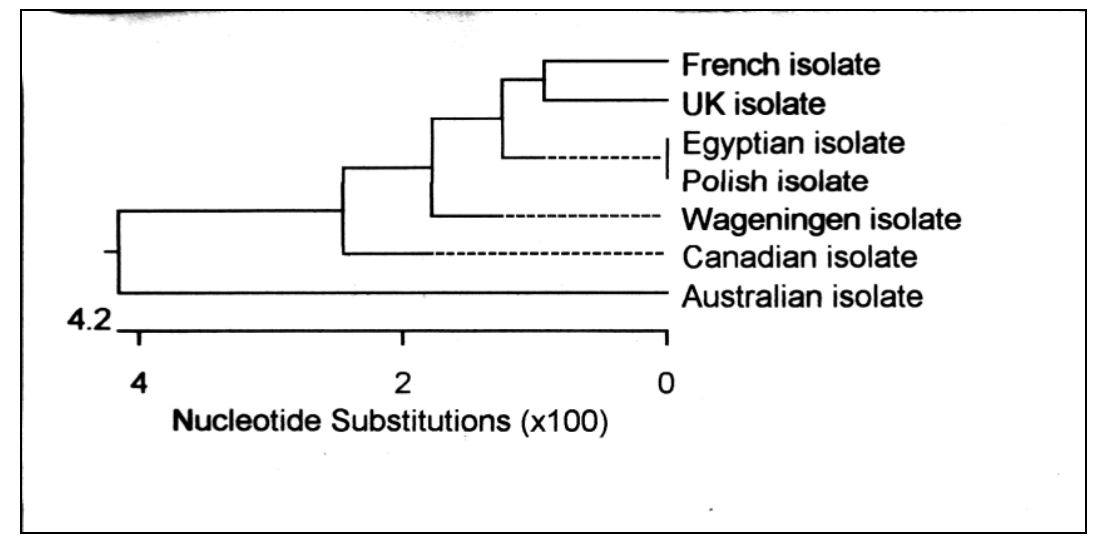

Table 2. Overall percent identity of PLRV- Egypt with different PLRV isolates in intergenic regions

\begin{tabular}{|l|c|}
\hline \multicolumn{1}{|c|}{ Strain } & Similarity \% \\
\hline Egypt X Australia & 90.5 \\
\hline Egypt X UK & 97.4 \\
\hline Egypt X Polish & 100 \\
\hline Egypt X French & 97 \\
\hline Egypt X Canada & 97.5 \\
\hline Egypt X Wageningen & 97.5 \\
\hline
\end{tabular}


Fig. 3. Phylogenetic tree determines dimensions of relationship between PLRV and different PLRV isolates in intergenic regions

\begin{tabular}{|c|c|c|c|c|c|c|c|c|c|}
\hline \multicolumn{10}{|c|}{ Percent Identity } \\
\hline \multirow{9}{*}{ 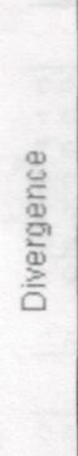 } & 1 & 2 & 3 & 4 & 5 & 6 & 7 & & \\
\hline & & 97.0 & 97.5 & 97.5 & 97.5 & 26.9 & 36.5 & 1 & Wageningen isolate \\
\hline & 3.1 & & 97.5 & 96.5 & 97.5 & 26.9 & 36.5 & 2 & Canadian isolate \\
\hline & 2.5 & 2.6 & & 97.0 & 100.0 & 26.9 & 35.5 & 3 & Egyptian isolate \\
\hline & 2.6 & 3.6 & 3.1 & & 97.0 & 26.4 & 36.5 & 4 & French isolate \\
\hline & 2.5 & 2.6 & 0.0 & 3.1 & & 26.9 & 35.5 & 5 & Polish isolate \\
\hline & 278.3 & 275.5 & 278.3 & 301.1 & 278.3 & & 25.4 & 6 & UK isolate \\
\hline & 140.6 & 140.8 & 147.6 & 140.8 & 147.6 & 350.0 & & 7 & Australian isolate \\
\hline & 1 & 2 & 3 & 4 & 5 & 6 & 7 & a & \\
\hline
\end{tabular}

Fig. 4A. Similarities between Luteoviruses RNA sequences in the intergenic regions, the sequence are following nucleotides 3368 (BWYV), 3473 (PLRV-EG) and 2745 (BYDV). Numbers in brackets are the length of intervening sequences in nucleotides, * indicates a match with PLRV-RNA sequences, - indicates that a gap has been inserted to enhance the degree of matching.

BWYV- GAUUAC-AAAUUCCUAGC-AGGCUUCG. (40) .UAUCUAUUCAUCUACC-U-AAGA (28). AUG

PLRV- GAUUAU-AAAUUUUUAGC-GGGAUUUG. (40) .UUUAGGAUUCTCATCC-U-AAGA (27). AUG

BYDV- UUACCAAAU-CUUAGCUGGG-UUG.. (44) ....UACUUUAUUUACAAUAAAGU (26). AUG

Fig. 4B. Sequence repeat in PLRV-RNA, the sequence start at 1577 (topleft) and ended at 1657 (bottom right). * indicated the same nucleotide in all three repeats. ! Indicates the same nucleotide in two of the three repeats

\author{
!*!********!*******!*!***!! \\ CCCGCAGAGAAAACUGCUCAAACAAAC \\ UCAGCAGAGAAGACUGCUCCAUCAACU \\ UCAGCAGAGAAAACUGCUCCAACAAAC
}

\title{
DISCUSSION
}

Number of different sources reported a very close values to our strain sequence as mentioned by Keese et al (1990) in Australia, Van der wilk et al., (1989) who determined the full genomic as 5882 nucleotide, while it was reported as 5885 nucleotides by Guyader and Durcray (2002). On the other hand, Mayo et al (1989) 
mentioned that 5987 nucleotide is the full genomic sequence of PLRV UK strain. All the sequences of mentioned strains are similar to the value of $6 \mathrm{~kb}$ estimated by Rowhani and Stace-Smith (1979) and $6.1 \mathrm{~kb}$ obtained by restriction mapping of cDNA. The putative $3^{\prime}$ terminal sequence is coterminal with that obtained by Prill et al (1988) and differs from that determined by Mayo et al (1989) strain in that our strain has C at position 5820 instead of $U$ at position 5871 instead of $G$ at position 5976 .

Comparing our results with those of Mayo et al (1989), similar base ratios were obtained ( $23 \% \mathrm{U}, 25 \% \mathrm{C}, 28 \% \mathrm{~A}$ and $24 \% \mathrm{G})$, of PLRV RNA Egypt, these results are similar to those of BWYV RNA and BYDV RNA (22\% U, 24\% C, $29.5 \%$ A and $24.5 \%$ G).

The sequence of PLRV Egyptian strain was compared the sequence of six different PLRV strains and the overall similarities for all genomic sequence are $100 \%, 98.5 \%$, 98.4\%, 98.3\%, 98\% and 93.5\% for polish, French, Wageningen (Dutch), UK, Canada, and Australia strains respectively. Tthese results are in harmony with those mentioned by Keese et al (1990) who compared the nucleotides sequences of an Australian and Canadian strains of PLRV Luteoviruses.

Results showed that five substantial open reading frames (ORFs) are present in PLRV RNA Egyptian strain. ORF1 started from nucleotide 70 till nucleotide 813 (AA1247), ORF2 from 203 to 2122 (AA1-639), ORF3 from 1540 to 3390 (AA 1-617), ORF 4 from 3588 to 5741 (AA1-718), while ORF5 started at 3613 and ended at 4083 (AA1156). In our sequence during cloning ORF4 it doesn't split into two ORFs as in case of those stains of (Guyader and Ducary, 2002 and Mayo et al 1989).

In other sequences and in our ORF4 (3588-4214) AA 1-208 and ORF 6 (42155741) AA (1-508), suggesting a conflict with the conceptual translation at amino acid 204. There are three non-coding regions in PLRV RNA. These are the $5^{\prime}$ terminal (70 nucleotides), the $3^{\prime}$ terminal (143 nucleotides) and 200 nucleotides between ORF3 and ORF4\&5. The intergenic regions present in our strain are similar to those reported by (Van der wilk et al 1989, and Guyader and Ducray, 2002).

There are also some similarities in the intergenic regions of PLRV RNA, and BWYV RNA and BYDV RNA (Veidt et al 1988). These similarities suggested that these stretches may be functionally significant. The right-hand stretch contain repeated UnA sequences followed by AAGA, two features suggested by Marsh et al (1988) to play a role in the formation of subgenmoic RNA. The abundant subgenomic fragments of PLRV RNA lies between nucleotides 4085 and 4795 or 4695 and 5196 approximately, so it may be subgenmoic mRNA for ORF4 sequence and sequence reported by Mayo et al (1989).

Ribosomal framshfiting can be used by different organisms to produce some kinds of protein form overlapping readingframes. This can be done in both directions. A shift in $3^{\prime}$ direction (+1) framshfit as mentioned by Bbelcourt and Farabaugh (1990). The 
other shift in $5^{\prime}$ direction (-1 framshift) has proven for Luteoviruses (Prufer et al., 1992, Garcie et al., 1993). In case of PLRV, the RNA dependent RNA polymerase is expressed by -1 ribosomal framshifting in the region of overlapping between ORF2 and $2 \mathrm{~b}$ as reported by Mayo et al (1989).

The signal responsible for efficient framshfiting in PLRV is composed of the slippery sequence uuuAAAu followed by a sequence that has the potential to adopt two alternative folding pattern, either a pseudoknot, or simple tem-loop structure as mentioned by Kujawa et al (1993) who confirmed that in PLRV-P, the -1 framshift in the overlap region depends on the slippery site and on the downstream positioned sequence. A proposed pseudoknot is needed for efficient framshifitng in the present study, the slippery sequences TTTAAAT (UuuAAAu) is located on position $1662 \mathrm{nt}$ in the overlapping region between ORF2 and ORF3. This result is in agreement with those reported for BWYV by Garcie et al (1993). In retrospect, could be concluded that the identified PLRV Egypt is $100 \%$ genetic identity with PLRV Polish.

\section{REFERENCES}

1. Beck, S. 1993. DNA Sequencing by Chemiluminescent Detection. In H. and A. Griffin (Ed.). Methods in Molecular Biology Vol. 23: DNA Sequencing Protocols. pp. 235-242. Humana Press Inc., Totowa, NJ.

2. Belcourt, M. F., and P. J. Farabaugh. 1990. Ribosomal frameshifting in the yeast retrotransposon TY. tRNA induce slippage on a 7 nucleotides minimal site. Cell b2: 339-352

3. Garcie, A., Duin, J. and Van Pleij, and C. W. A. 1993. Differentional response to frameshift signal in Eukaryotic and Prokaroytic translational systems. Nucl. Acids Res. 21:401-406

4. Gamal Eldin, A. S., A. A. Sallam., H. M. Abdelmaksoud, and E. K. Fahimy. 2004. Complete Nucleotide Sequence of Potato leaf roll virus infects Potato in Egypt. Abst. (83) World Potato Congress, Kunming - China

5. Guyader, S. and D. G. Ducray. 2002. Sequence analysis of potato leaf roll virus isolates reveals genetic stability, major overlapping events and differential selection pressure between overlapping reading frame products. J. Gen. Virol. 83 (pt 7): 1799-1807

6. Keese, P., R. R. Martin, L. M. Kawchuk, P. M. Waterhouse and W. L. Gerleach. 1990. Nucleotide sequence of an Australian and Canadian isolate of potato leaf roll Luteeovirus and their relationship with two European isolates. J. Gen. Virol. 71 (pt 3): 719-724

7. Kujaw, A. B., G. Drugeon, D. Hulanicke and A. L. Haenni. 1993. Structural requirments for efficient translational frameshifitng in the synthesis of the 
putative viral RNA dependent RNA polymerase of potato leaf roll virus. Nucl. Acids Res. 21(a): 2185-2171

8. Marsh, L. S., T. W. Dreher and T. C. Hall. 1988. Mutational analysis of the care and modulator sequence of the BMV RNA3 subgenomic promoter. Nucl. Acids Res. 1: 1385-1397

9. Palucha, A., E. Sadowy, A. Kujawa, M. Juszczuk, W. Zagorski, and D. Hulanicka. 1994. Nucleotide sequence of RNA of polish isolate of potato leaf roll Luteovirus. Acta Biochim. Pol. 41 (4): 405-414

10. Prufer, D., E. Tacke, J. Schmitz, B. Kull, A. Kaufmann, and W. Rodhe. 1992. Ribosomal frameshifitng in plants: a noval directs the -1 frameshift in the synthesis of the putative viral replicase of poato leaf roll Luteovirus. EMBO J. 11 (3): 1111-1117

11. Sangar, F., S. Nicklen and A.R. Coulson. 1977. DNA sequencing with chain terminating inhibitors. Proc. Natl. Acad. Sci. USA 74, 5463-546

12. Sambrook, J., E. F. Fritsch and T. Maniatis. 1989. Molecular cloning: A laboratory manual. Cold Spring Harbor Laboratory press, cold Spring Harbor. New York.

13. Rowhani, A., and R. Stace-Smith. 1979. Purification and Characterization of Potato leaf roll virus. Virology 98: 45-54

14. Waterhouse, P. M., F. E. Gildow and G. R. Johnstone. 1988. Luteovirus group. AAB Descriptions of Plant Viruses No. 339 
التعريف الجزيئي والتحليل الجيني السلالي لفيروس التفاف اوراق البطاطس في مصر هشام محدين عبد المقصود، ايمن محد مندور، احمد شوقى جمال الدين

$$
\text { قسم بحوث الفيروس - معز بحوث امراض النباتات - مركز البحوث الزراعية - الجيزة }
$$

تم عزل فيروس التفاف الاوراق في البطاطس من حقول البطاطس المصرية وتم تعريفة على الإنى الإني

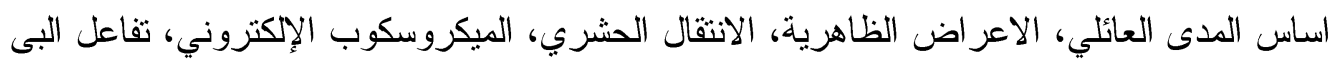

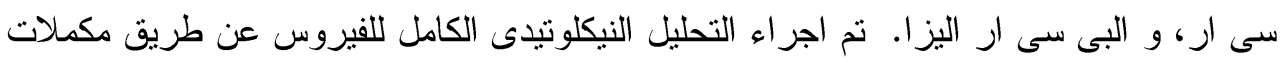

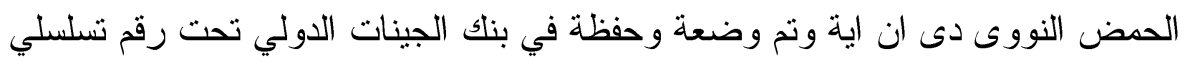

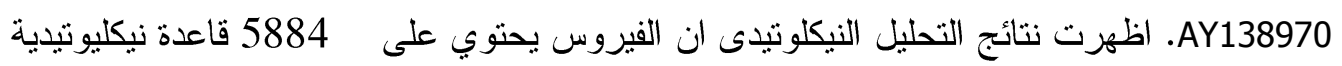

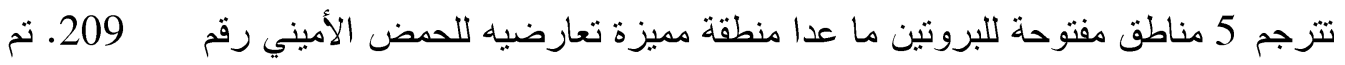

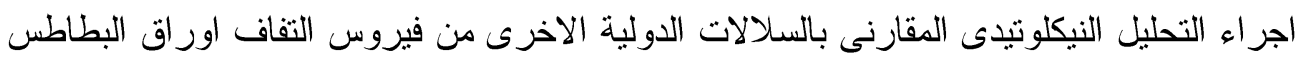

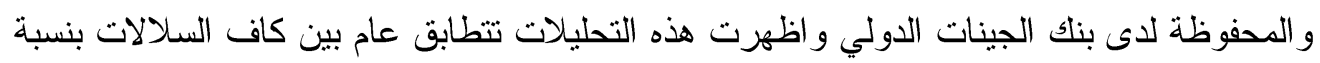
97.02 وطابق خاص بكل سلالة دولية كتالي 100\% (السلالة البولندية)، 98.5\% (السلالة

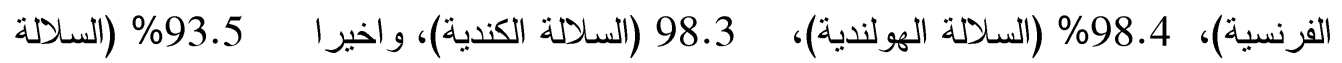

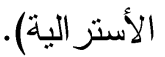

